\title{
Formation of polymer nanoparticles by UV pulsed laser ablation of poly (bisphenol A carbonate) in liquid environment
}

\author{
Daniel E. Martínez-Tong ${ }^{1,+}$, Mikel Sanz ${ }^{2}$, Tiberio A. Ezquerra ${ }^{1}$, Aurora Nogales ${ }^{1}$, J.F. \\ Marco $^{2}$, Marta Castillejo ${ }^{2}$, Esther Rebollar*2 \\ ${ }^{1}$ Instituto de Estructura de la Materia, IEM-CSIC, Serrano 121 \\ ${ }^{2}$ Instituto de Química Física Rocasolano, IQFR-CSIC, Serrano 119, 28006, Madrid \\ ${ }^{+}$Present address: Donostia International Physics Center (DIPC) \& Centro de Física de \\ Materiales (CSIC-UPV/EHU), P. M. de Lardizabal 5, 20018 San Sebastián, Spain. \\ *E-mail:e.rebollar@csic.es
}

Suspensions of poly(bisphenol A carbonate) (PBAC) nanoparticles of varying size and shape have been produced by ablation of a PBAC target in liquid media with the fourth harmonic of a Q-switched Nd:YAG laser (wavelength $266 \mathrm{~nm}$, full width at half maximum $4 \mathrm{~ns}$, repetition rate $10 \mathrm{~Hz}$ ). The polymer target was placed at the bottom of a rotating glass vessel filled with around a $10 \mathrm{~mm}$ column of liquid. Laser ablation in water leads to spherical nanoparticles with diameters of several tens of nanometers for fluences close to $1 \mathrm{~J} / \mathrm{cm}^{2}$. Ablation at lower fluences, around $0.1 \mathrm{~J} / \mathrm{cm}^{2}$, results in the production of nanoparticles of smaller diameters and also of non-spherical nanoparticles. Additional irradiations at the fluence of $0.1 \mathrm{~J} / \mathrm{cm}^{2}$ were performed in several liquid media with different properties, in terms of density, viscosity, thermal conductivity, boiling temperature, isothermal compressibility and polarity. The different size distributions observed were related to the thermal conductivity of the systems, while their viscosity seems to be responsible for the development of nanostructures with different morphologies.

Keywords: pulsed laser ablation in liquids, polymer ablation, polymer nanoparticles 


\section{Introduction}

Interest in the properties of polymers confined into the nanometer scale is increasing in recent years [1]. Polymers are widely used in nanofabrication processes like wires of nanometer-scale diameters [2], nanoscale polymeric particles [3, 4] and nanoimprinting [1].

Polymer nanoparticles have found use in a number of applications like coating and functionalization of surfaces [5], adhesives [6], drug delivery systems [7], enhanced polymer blends preparations [8], organic photovoltaic systems [9] and smart/stimuliresponsive materials [10]. The applications of polymer nanoparticles are significantly affected by their physical properties as well as their surface morphology. Both factors can be controlled by the preparation method used to generate such particles.

Different methods for preparing polymer nanoparticles have been proposed [11-13]. Insitu polymerization can be carried out so that from a monomer it is possible to obtain a nanostructured polymer in the form of a particle [12]. A different approach consists of the use of a previously synthesized polymer in solution and the subsequent processing of the material to generate the nanostructures $[4,13,14]$.

Pulsed laser ablation has evolved as one of the most efficient physical methods for nanofabrication [15, 16]. In particular, pulsed laser ablation of solids in liquid environment (PLAL) was reported for the first time in 1987 [17]. In that work, a metastable phase of iron oxide was synthesized by ablating an iron target in water. Since then, this technique has been extensively investigated for the s fabrication of nanoparticles of a large variety of materials, including metals, alloys, ceramics and semiconductors [1725]. More recently such approach has also been proposed to process insoluble organic compounds, like phtallocyanines [26, 27], aromatic hydrocarbons [27] and polymers [28]. Remarkable advantages of this method over chemical synthesis rely on the simplicity of the procedure, the weak aggregation effects, and the lack of impurities caused by chemical precursors. The nanoparticle concentration, size, polydispersity, shape, and solid phase (amorphous or crystalline) can be controlled by the adequate choice of laser wavelength, pulse duration, and fluence $[18,23,29,30]$.

In the case of PLAL of polymeric materials, nanoparticles of several polymers such as poly(ethylene terephthalate) (PET), polycarbonate (PC), polyimide (PI) and polystyrene (PS) have been obtained in water [28] upon ablation at $248 \mathrm{~nm}$. However the role of the 
liquid media on the characteristics of the particles obtained by PLAL has not been described in detail. In the present work we present results on PLAL of poly (bisphenol A carbonate) (PBAC) in several liquid media paying special attention to their different properties in terms of density, viscosity, thermal conductivity, boiling temperature, isothermal compressibility and polarity.

\section{Experimental}

As received poly (bisphenol A carbonate) (PBAC) (Lexan ML3021A, SABIC I-P (Innovative Plastics) was dried in a vacuum oven for 16 hours at $100{ }^{\circ} \mathrm{C}$. After drying PBAC pellets were heated in a thermoplastic press at $220^{\circ} \mathrm{C}$ for 2 minutes and a pressure of 20 bar was applied for 3 minutes. Then, the film was cooled down, inside the press using cold cartridges. The resulting PBAC films had a thickness of $0.9 \pm 0.1 \mathrm{~mm}$ and were cut into disks with a diameter of $0.9 \mathrm{~cm}$.

Ablation of PBAC targets was performed with the fourth harmonic (266 nm) of a Qswitched Nd:YAG laser (Quantel Brilliant B, 5 ns pulse duration FWHM) operating at a repetition rate of $10 \mathrm{~Hz}$. The experimental set-up is similar to the one reported in $[23,31]$. In brief, the PBAC target was placed at the bottom of a glass vessel filled with a ca. 10 $\mathrm{mm}$ column $(1.0 \mathrm{~mL})$ of different solvents. The laser beam was focused by a $12.5 \mathrm{~cm}$ focal length lens situated approximately at $10 \mathrm{~cm}$ from the target to achieve a spot diameter of $1 \mathrm{~mm}$ on its surface. The diameter of the laser spot was measured from the trace left by the laser pulse on an uncoated PVC plate. The vessel was rotated at about 10 rpm during ablation to avoid target cratering. Several laser fluences in the range 0.1- 1 $\mathrm{J} / \mathrm{cm}^{2}$ were employed and total irradiation time was 1 hour (36000 pulses).

As liquid media we used six different liquids: 1$)$ deionized water $\left(\mathrm{H}_{2} \mathrm{O}\right)$, purified using a Milli-Q 18.2 M $\Omega$ reagent-grade water system from Millipore, 2) 2-propanol (2P) (99.98\%, Aldrich), 3) n-hexane (HEX) (HPLC grade, Scharlau), 4) heptane (HEP) (99\%, Aldrich), 5) carbon tetrachloride $\left(\mathrm{CCl}_{4}\right)(99 \%$, Panreac) and 6) ethylene glycol (99\%, EG) (Panreac). Some pertinent physical magnitudes of every liquid are listed in Table 1 . The solubility was tested in a macroscopic solubility experiment by immersing $24 \mathrm{mg}$ of PBAC in $2 \mathrm{~mL}$ of the different liquids. In all the cases PBAC was not soluble except for carbon tetrachloride. In this case, and for time intervals comparable to the experimental 
time used in the PLAL protocol, the PBAC target did not show any changes by eye inspection. However, after a few days, swelling and whitening of PBAC was observed.

UV-Vis absorption spectra of all the liquids employed were recorded with a UV-Vis-NIR spectrophotometer (UV-3600 Shimadzu) using $1 \mathrm{~cm}$ optical path quartz cuvettes. Figure 1 shows the absorption spectra collected in the $200-300 \mathrm{~nm}$ range. It is evident that at the irradiation wavelength of $266 \mathrm{~nm}$, absorption of the liquids employed is negligible except for carbon tetrachloride which absorbs slightly more.

Table 1. Density $(\rho)$, boiling temperature $\left(T_{b}\right)$, thermal conductivity $(k)$, viscosity, isothermal compressibility, polarity (normalized $\mathrm{E}_{\mathrm{T}}{ }^{\mathrm{N}}$ values) and solubility of PBAC on the different liquids employed. Size of the PBAC particles obtained upon irradiation at $0.1 \mathrm{~J} / \mathrm{cm}^{2}$ is also listed.

\begin{tabular}{|c|c|c|c|c|c|c|}
\hline Liquid & $\begin{array}{l}\text { n- } \\
\text { hexan } \\
\text { e }\end{array}$ & Heptane & $\begin{array}{l}\text { Distilled } \\
\text { water }\end{array}$ & $\begin{array}{l}\text { Carbon } \\
\text { tetrachloride }\end{array}$ & $\begin{array}{l}2- \\
\text { propanol }\end{array}$ & $\begin{array}{l}\text { Ethylene } \\
\text { glycol }\end{array}$ \\
\hline$\rho\left(\mathrm{g} / \mathrm{cm}^{3}\right)$ & 0.66 & 0.68 & 1 & 1.6 & 0.79 & 1.1 \\
\hline$T_{b}\left({ }^{\circ} \mathrm{C}\right)$ & 68 & 98 & 100 & 77 & 82 & 197 \\
\hline$k(\mathrm{~W} / \mathrm{m} \mathrm{K})[32]$ & 0.120 & 0.123 & 0.606 & 0.103 & 0.141 & 0.254 \\
\hline $\begin{array}{l}\text { Viscosity } \\
(\mathrm{g} / \mathrm{m} \mathrm{s})[33]\end{array}$ & $\begin{array}{l}0.294 \\
2\end{array}$ & 0.3967 & 0.8909 & 0.9004 & 2.0436 & 13.8 \\
\hline $\begin{array}{l}\text { Isothermal } \\
\text { Compressibility } \\
\left(\text { at } 20^{\circ} \mathrm{C}\right)\left(10^{-}\right. \\
\left.{ }^{-} / \mathrm{MPa}^{-1}\right)[34]\end{array}$ & 16.69 & 14.38 & 4.59 & 10.50 & 13.32 & 3.64 \\
\hline Polarity [35] & 0.009 & 0.012 & 1 & 0.052 & 0.546 & 0.79 \\
\hline $\begin{array}{l}\text { PBAC } \\
\text { solubility }\end{array}$ & $\begin{array}{l}\text { Non- } \\
\text { solubl } \\
\text { e }\end{array}$ & $\begin{array}{l}\text { Non- } \\
\text { soluble }\end{array}$ & $\begin{array}{l}\text { Non- } \\
\text { soluble }\end{array}$ & Swelling & $\begin{array}{l}\text { Non- } \\
\text { soluble }\end{array}$ & $\begin{array}{l}\text { Non- } \\
\text { soluble }\end{array}$ \\
\hline $\begin{array}{l}\text { PBAC particle } \\
\text { size }(n m)\end{array}$ & $26 \pm 3$ & $27 \pm 3$ & $18 \pm 2$ & $40 \pm 5$ & $29 \pm 3$ & $25 \pm 3$ \\
\hline
\end{tabular}




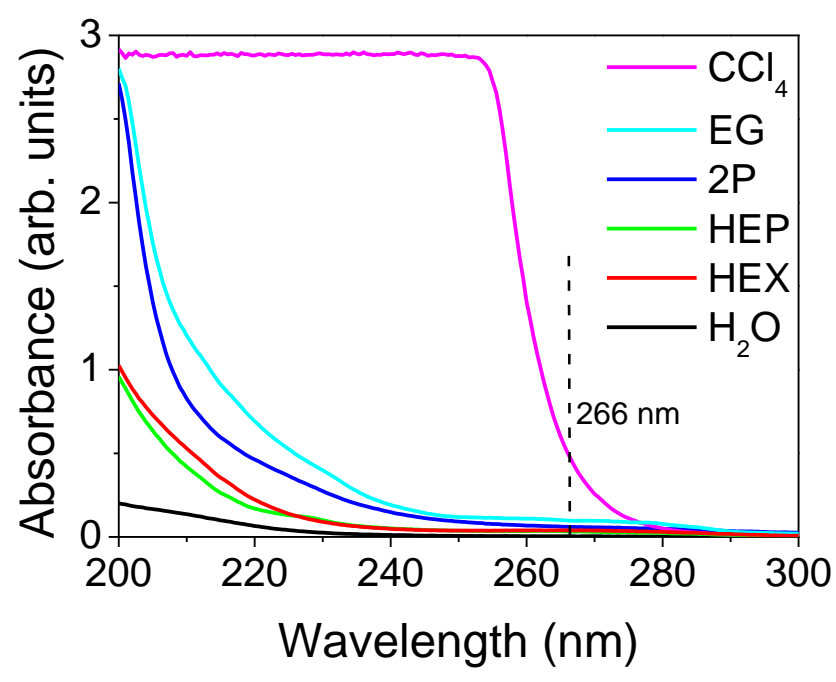

Figure 1. Optical absorption spectra of the different liquids used in PLAL experiments. The dashed line indicates the laser irradiation wavelength of $266 \mathrm{~nm}$.

Size analysis of the obtained nanoparticles was performed by Atomic Force Microscopy, AFM (Multimode 8 equipped with a Nanoscope V controller, Bruker) in tapping mode, using NCHV probes (Bruker). In all the cases, $20 \mu \mathrm{L}$ of the resulting suspensions were drop-casted on pieces of silicon wafer (100). Samples were left drying for ca. 20 hours at ambient conditions, except for the case of ethylene glycol, which was dried under vacuum at a temperature of $30^{\circ} \mathrm{C}$. Images were analysed with the software Nanoscope Analysis 1.50 (Bruker). For the nanoparticles size study five independent images were recorded and analysed. X-ray photoelectron spectroscopy (XPS) data were acquired using $\mathrm{Al} \mathrm{K}_{\mathrm{a}}$ radiation $(\mathrm{hn}=1486.6 \mathrm{eV})$. All reported spectra were recorded at an electron take-off angle of $90^{\circ}$ with a constant analyzer pass energy of $20 \mathrm{eV}$. All binding energies are referred to the $\mathrm{Ru} 3 \mathrm{~d} 5 / 2$ signal, which was set to $280.0 \mathrm{eV}$. The spectra were fitted with pseudo Voigt line profiles and a Shirley background using the CasaXPS software (version 2.3.16).

\section{Results}

\subsection{Dependence on laser fluence}

Figure 2 shows AFM topography images of nanoparticles obtained in water upon irradiation at $266 \mathrm{~nm}$ and 3600 pulses at different fluences. For irradiation at fluences of 1 and $0.4 \mathrm{~J} / \mathrm{cm}^{2}$ the deposited material is mainly constituted by round nanoparticles. 
Additionally, some larger aggregates are observed which can be related to possible agglomeration of material [36]. For lower fluences of 0.2 and $0.1 \mathrm{~J} / \mathrm{cm}^{2}$ the resulting morphology follows a similar behavior, and spherical nanoparticles with smaller diameters are observed together with different morphologies, specifically strand-like structures which will be described below.
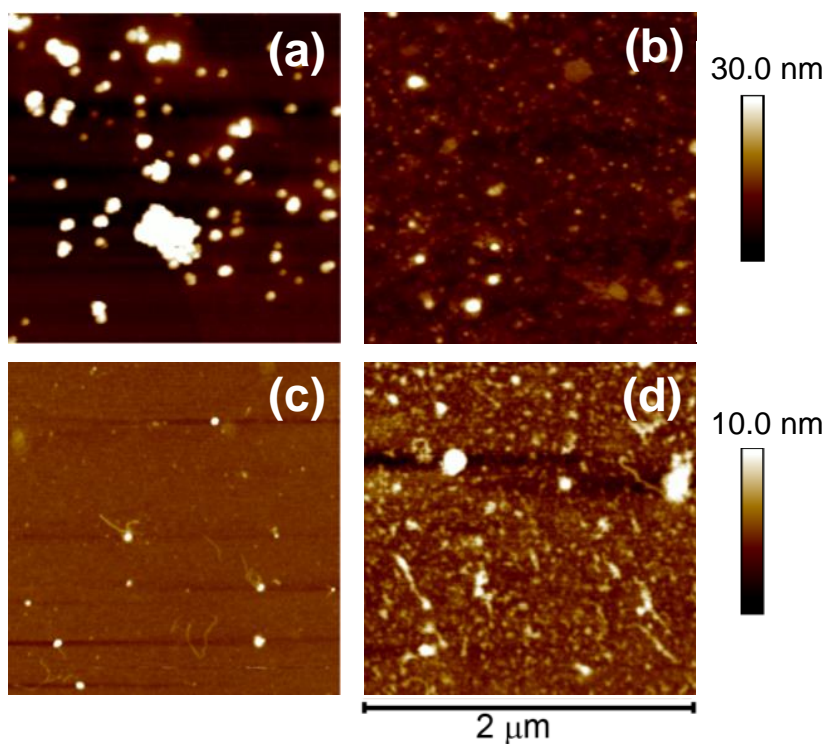

Figure 2. Height AFM images of PBAC nanoparticles formed in water at (a) $1 \mathrm{~J} / \mathrm{cm}^{2}$, (b) $0.4 \mathrm{~J} / \mathrm{cm}^{2}$, (c) $0.2 \mathrm{~J} / \mathrm{cm}^{2}$ and (d) $0.1 \mathrm{~J} / \mathrm{cm}^{2}$.

The size distribution and average diameter of nanoparticles obtained from the AFM images are shown in Figure 3. For irradiation at fluences of 1 and $0.4 \mathrm{~J} / \mathrm{cm}^{2}$ the mean diameters are $54 \pm 10 \mathrm{~nm}$ and $48 \pm 10 \mathrm{~nm}$ respectively. Aggregates had not been taken into consideration for the statistical size distribution analysis.

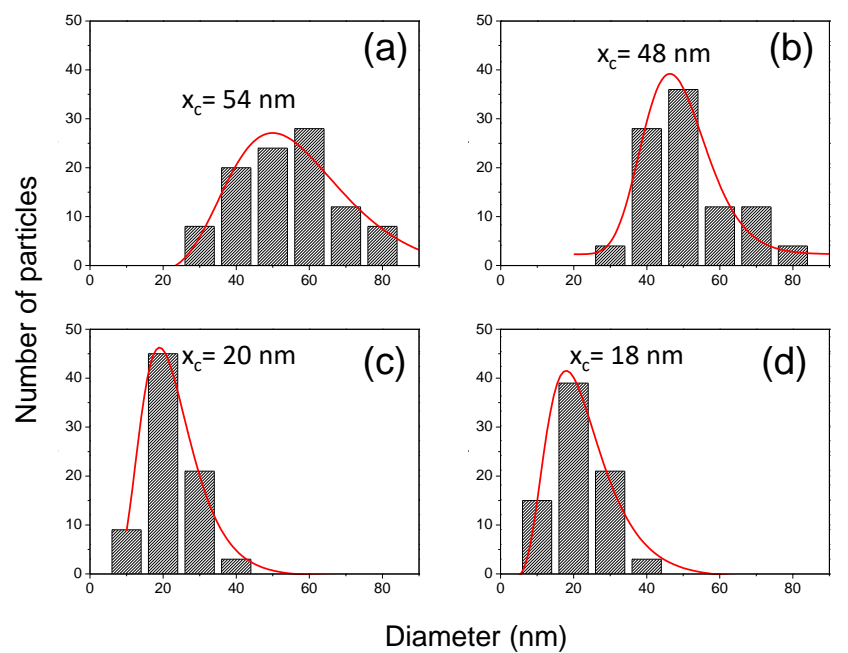


Figure 3. Size histograms of the PBAC nanoparticles obtained in water at (a) $1 \mathrm{~J} / \mathrm{cm}^{2}$, (b) $0.4 \mathrm{~J} / \mathrm{cm}^{2}$, (c) $0.2 \mathrm{~J} / \mathrm{cm}^{2}$ and (d) $0.1 \mathrm{~J} / \mathrm{cm}^{2}$. Lines are fits to the lognormal distribution function.

For lower irradiation fluences of 0.2 and $0.1 \mathrm{~J} / \mathrm{cm}^{2}$ the mean diameters are $20 \pm 5$ and 18 $\pm 5 \mathrm{~nm}$ respectively. Figure 4 shows the variation of the mean diameter as a function of fluence for spherical nanoparticles. It is observed that nanoparticle mean size increases as fluence increases. Also, it seems that this variation follows a step-like trend, indicating that there is a critical fluence at which an increase in size occurs quite suddenly.

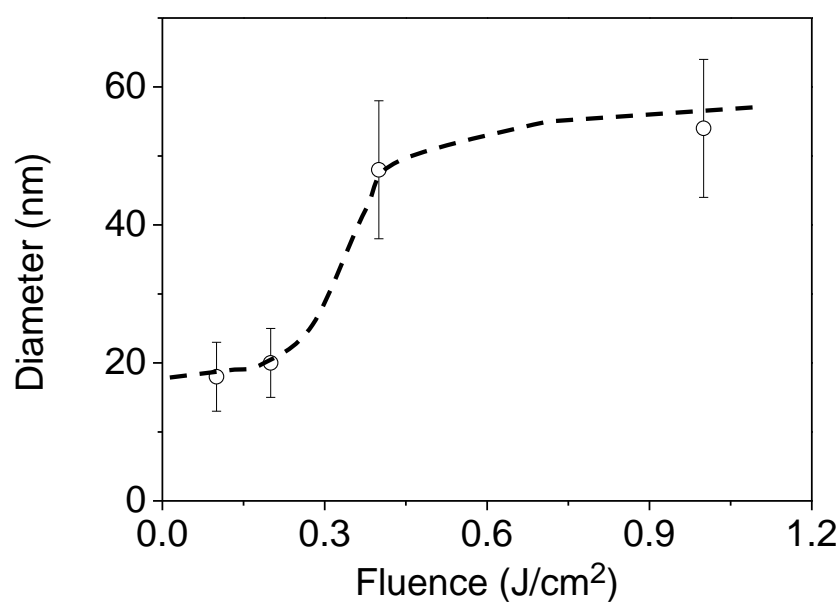

Figure 4. Mean diameter of spherical nanoparticles of PBAC in water as a function of fluence in water. The dashed line is shown as a visual guide.

In addition to the decrease of nanoparticle diameter when decreasing the ablation fluence another interesting observation is the appearance of different morphologies, specifically strand-like structures (see Figure 2c and 2d). Figure 5 shows enlarged AFM topography images of some structures obtained at $0.2 \mathrm{~J} / \mathrm{cm}^{2}$. The height of these strands typically lies between $1-3 \mathrm{~nm}$ with lengths in the range of $100-450 \mathrm{~nm}$.
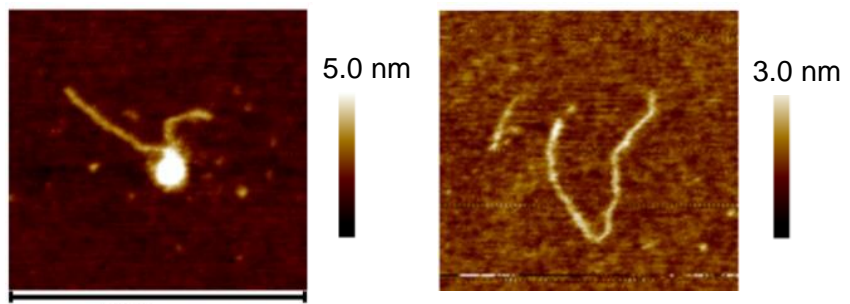

$500 \mathrm{~nm}$ 
Figure 5. Detailed height AFM images of nanostructured material formed by PLAL in water of PBAC at $0.2 \mathrm{~J} / \mathrm{cm}^{2}$.

XPS analysis of the material obtained at the lowest fluence employed, ie. $0.1 \mathrm{~J} / \mathrm{cm}^{2}$, was performed and the obtained spectra are shown in Figure 6. To fit the PBAC spectrum, the C 1s spectra can be represented by five components which correspond to different bonding states. In particular, $284.3 \mathrm{eV}$ corresponds to aromatic $\mathrm{C}-\mathrm{H}, 285.1 \mathrm{eV}$ to aliphatic $\mathrm{C}-\mathrm{H} / \mathrm{C}-\mathrm{C}, 286.3 \mathrm{eV}$ to aromatic $\mathrm{C}-\mathrm{O}, 288.8 \mathrm{eV}$ to $\mathrm{O}-\mathrm{C}=\mathrm{O}$, and $290.4 \mathrm{eV}, \mathrm{O}-\mathrm{C}-\mathrm{O}$ [37]. The contribution of each one is listed in Table 2. In the case of nanoparticles obtained by PLAL there is a slight increase of the signal corresponding to $\mathrm{O}-\mathrm{C}=\mathrm{O}$. The formation of new functional groups such as ketones, aldehydes, acid and ester groups, it has been reported previously for irradiation of polymers [38, 39] or other treatments for instance by plasma [37]. Another change observed is the increase of the ratio aromatic carbon/aliphatic carbon, which could be related to the oxidation of aliphatic methyl carbons.

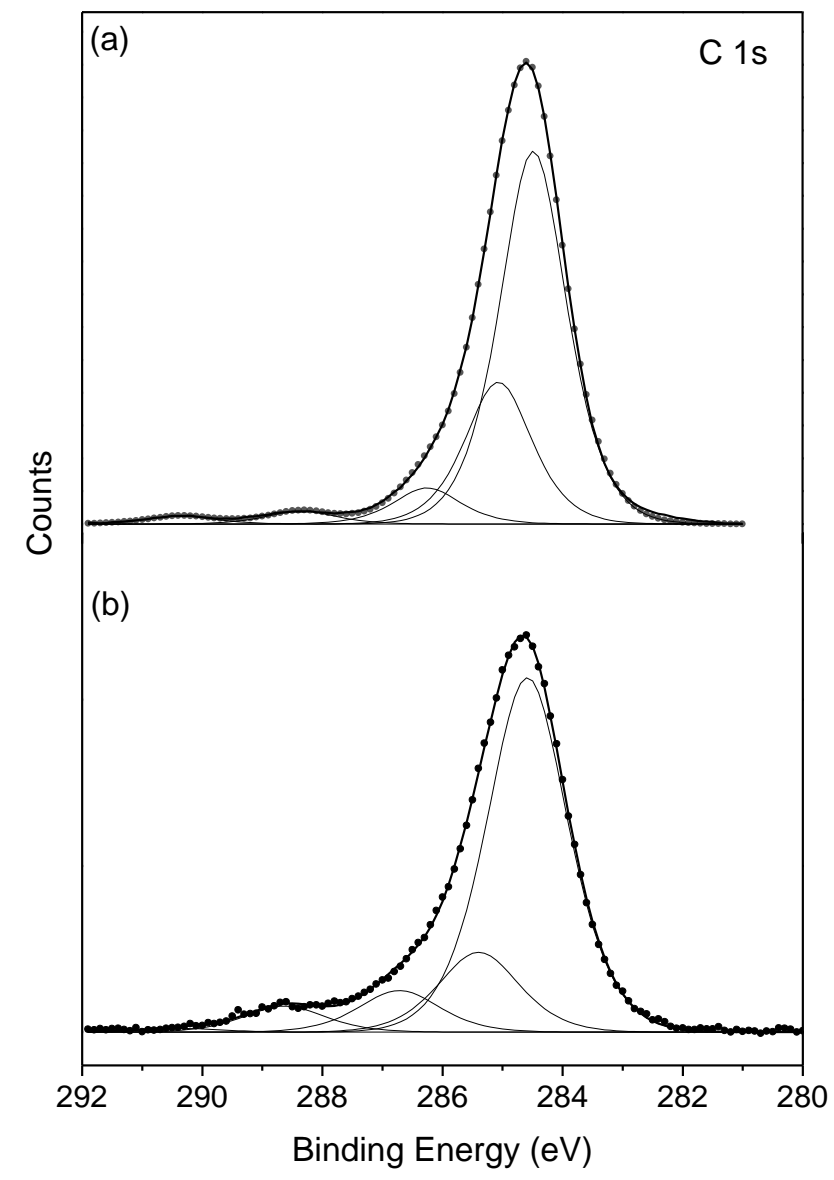


Figure 6. C 1s XPS spectra from (a) PBAC target and (b) PBAC nanoparticles obtained by PLAL at $0.1 \mathrm{~J} / \mathrm{cm}^{2}$. In both, the experimental data are shown with circles, components used in the fit are marked by thin lines and the resulting fit by thick lines.

Table 2. Data from curve fitting of spectra displayed in Figure 5.

\begin{tabular}{|c|c|c|c|c|c|c|}
\hline Sample & C aromatic & C aliphatic & $\begin{array}{c}\text { C-O } \\
\text { aromatic } \\
\text { C=O }\end{array}$ & $\begin{array}{c}\text { O-C-O } \\
\text { O-C=O }\end{array}$ & $\begin{array}{c}\text { C aromatic / } \\
\text { C aliphatic }\end{array}$ \\
\hline PBAC & 65 & 25 & 6 & 2 & 2 & 2.6 \\
\hline $\begin{array}{c}\text { PBAC } \\
\text { PPs }\end{array}$ & 70 & 16 & 8 & 1 & 5 & 4.4 \\
\hline
\end{tabular}

\subsection{Dependence on the liquid media}

In PLAL experiments the liquid environment confines the expansion of the plasma plume. Thus, the physico-chemical properties of a specific liquid might influence the interaction with the ablation plume [40, 41]. In order to address this point, we performed PLAL experiments in different liquid media whose properties are described in Table 1. The experiments were carried out at a fluence of $0.1 \mathrm{~J} / \mathrm{cm}^{2}$ and 36000 pulses. Figure 7 shows height AFM images of the different samples. An image of the irradiation in water is also included for comparison, in which the elongated nanostructures, described in Figures 2 and 5, are also observed. Some of these elongated structures were also observed for irradiation in carbon tetrachloride (Figure 7b) and n-hexane (Figure 7c).

In carbon tetrachloride isolated spherical and elongated nanoparticles, as well as round particles with attached strands are observed. Although these results qualitatively resemble those obtained in water, the sizes of the nanostructures are different. In carbon tetrachloride, the strands emerging from the spherical particles have heights between 10 $-20 \mathrm{~nm}$ and lengths between $100-200 \mathrm{~nm}$. 

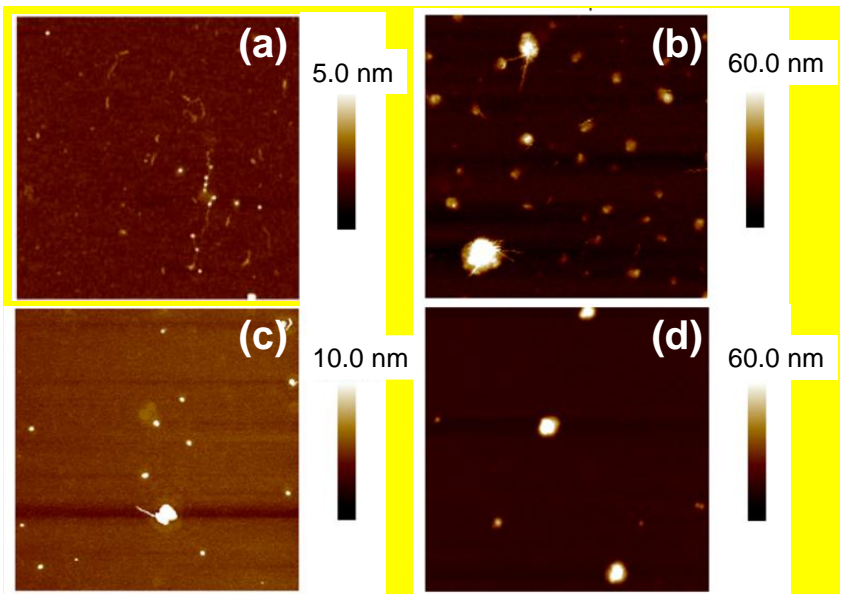

$60.0 \mathrm{~nm}$
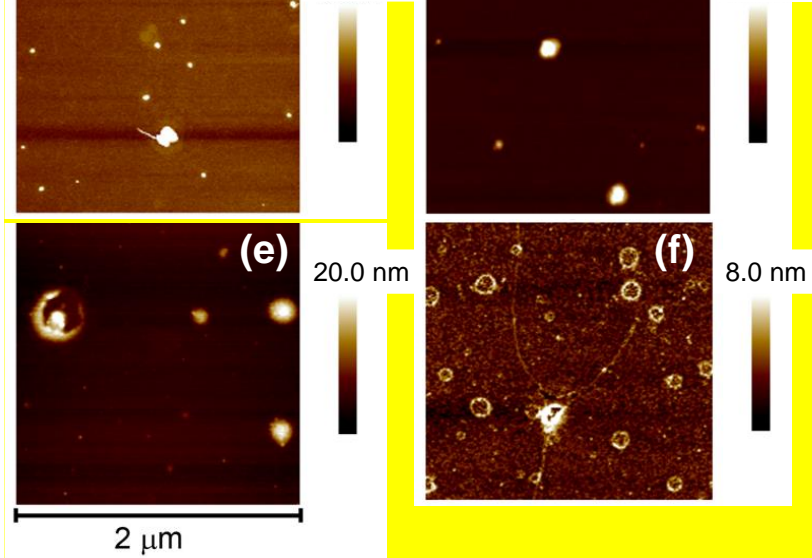

$8.0 \mathrm{~nm}$

Figure 7. Height AFM images showing in detailed the nanostructures formed by irradiation at an ablation fluence of $0.1 \mathrm{~J} / \mathrm{cm}^{2}$ in (a) water, (b) carbon tetrachloride, (c) nhexane, (d) heptane, (e) 2-propanol and (f) ethylenglycol.

Figure 8 shows the size distributions of the spherical particles obtained by PLAL in the different liquids, and the mean diameters are listed in Table 1. For carbon tetrachloride (Figure 7b), the spherical particles that serve as cores for the strands present a wide range of diameters, from $20-80 \mathrm{~nm}$, having a mean size of $40 \mathrm{~nm}$. Nanoparticles obtained in n-hexane (Figure 7c) feature a mean diameter of $26 \mathrm{~nm}$ and the observed strands have diameters and lengths in the same range as the ones observed for irradiation in carbon tetrachloride. However, in this case the amount of nanoparticles is higher than the amount of strands. For the rest of the liquids tested, the presence of these elongated nanostructures is not detected. In the case of heptane, only spherical particles are observed with a mean diameter of about $27 \mathrm{~nm}$ (Figure 7d).

AFM images of the particles prepared in 2-propanol show two different size distributions (see Figures 7e and 8e). Smaller particles have a mean diameter of $29 \mathrm{~nm}$, similar to those obtained for irradiation in heptane, while larger particles have mean diameters of about $120 \mathrm{~nm}$. Additionally, as can be seen in Figure 7e, a new feature is revealed, consisting of a toroid structure surrounding a particle, the latter appearing to be physically attached 
to the toroid through one of its sides. The toroids observed for irradiation in 2-propanol are not fully closed and have thicknesses and diameters around 10 and $350 \mathrm{~nm}$ respectively. This kind of structures is reproducible and observed in several zones of the deposited samples.

Finally, in the case of irradiation in ethylene glycol a continuum of nanoparticles, without any specific geometry appears throughout the entire AFM image (Figure 7f). The roughness of this phase is about $1 \mathrm{~nm}$, which is much higher than the underlying silicon substrate. Some spherical particles can be also observed in the continuum layer, having mean diameters of $25 \mathrm{~nm}$. However, in this case the most important features are toroids, as observed for irradiation in 2-propanol. These toroids seem to emerge from the continuum, having thicknesses between $1-5 \mathrm{~nm}$ and mean diameters of about $140 \mathrm{~nm}$. Additionally, strands can be seen in the AFM image with thicknesses of around $1 \mathrm{~nm}$ and lengths in the micrometer scale.

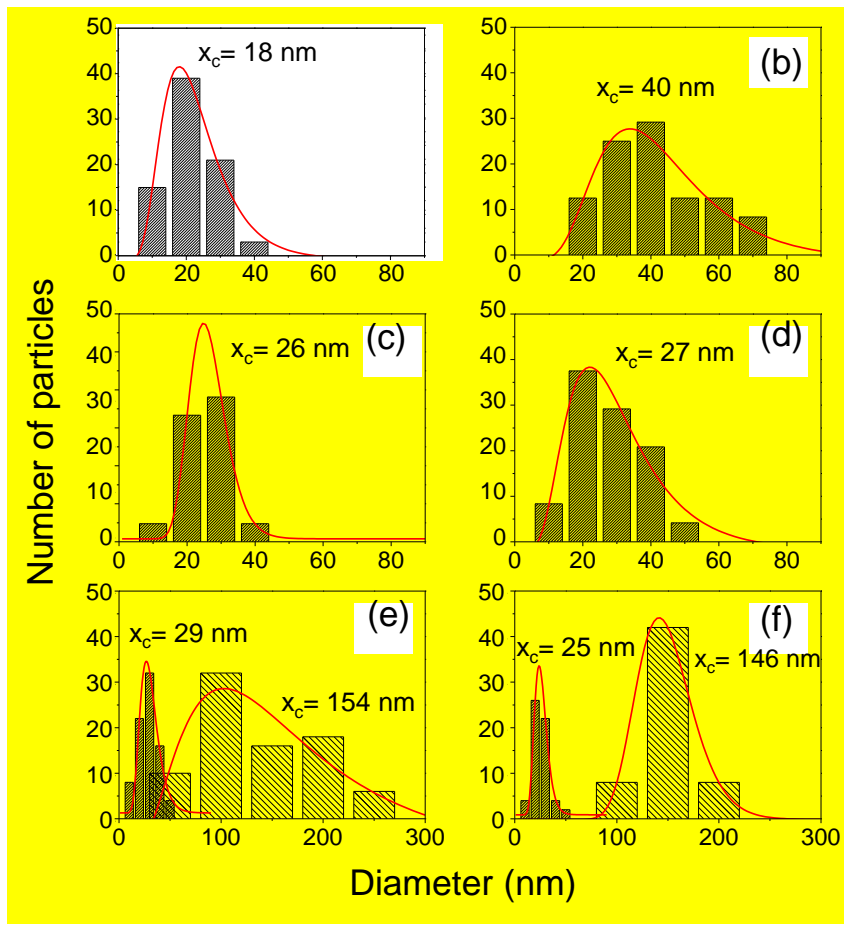

Figure 8. Size distribution histograms of nanostructures obtained in (a) water, (b) carbon tetrachloride, (c) n-hexane, (d) heptane, (e) 2-propanol and (f) ethylenglycol. Lines correspond to the lognormal fitting.

\section{Discussion}


Irradiation of PBAC in a liquid environment at fluences above the polymer ablation threshold induces the ejection of material and the generation of a plasma plume. As material is ejected from the polymer target, the liquid phase provides efficient thermal quenching. Also, the liquid confines the evolution of the plasma plume [22, 42].

From the results shown above it is clear that there is a dependence of the nanoparticle formation, in terms of size and shape, both on the laser fluence and on the liquid media. The different size distributions and types of structures as the ablation fluence is varied in the range of 0.1 to $1 \mathrm{~J} / \mathrm{cm}^{2}$ are related to the fact that material can be ejected from deeper layers as the fluence increases, thus higher amount of material is ejected. For lower fluences, the reduction of size and the appearance of new geometrical structures might indicate that the ablated species experience a higher degree of interaction with the liquid surroundings. In the PLAL setup, as the laser beam heats the PBAC target, the liquid environment is also affected. Previously, Leveugle and Zhigilei [43] have shown, via molecular dynamics simulations, that during the ablation process, the original randomly oriented molecules in the target have a tendency to extend along the flow in the ablation plume and to thread through the liquid regions. In their simulations, the polymer-liquid environment leads to the formation of complex polymer elongated structures in the direction of the ablation plume expansion [43]. Also, these authors have shown a dependence of this process with the laser fluence. For low fluences, elongated polymer structures appear, while as fluence is increased, the material was ejected as clusters [43, 44]. These facts can be compared to the results shown herein. Also, at high fluences, the amount of ejected material is higher and is ejected with a higher kinetic energy in comparison to the low fluence ablation. This might induce more collisions in the ablation plume that ultimately lead to coalescence of material and thus to higher diameters of the resulting nanoparticles, as observed.

The shape of the observed nanostructures is shown to be highly dependent on the liquid environment, and nanospheres, strands, cylinders and toroids have been observed by AFM. In a first approach this could be related both to different mechanisms of molecular ejection and to the different physical properties of each liquid (Table 1). As the PBAC target is being ablated, the high temperatures at the target surface lead to the formation of bubbles due to the evaporation of the solvent. These bubbles could be linked to the formation of the different nanoparticle structures. According to Lazare [45], the temperature increase $(\Delta T)$ at the target surface can be estimated as $\Delta T=\alpha F /\left(\rho C_{p}\right)$ where $F$ 
is the laser fluence, $\alpha$ is the absorption coefficient at the irradiation wavelength of 266 $\mathrm{nm}\left(18000 \mathrm{~cm}^{-1}\right), \rho$ is the density $\left(1200 \mathrm{~kg} / \mathrm{m}^{3}\right)$ and $C_{p}$ is the heat capacity $(1207 \mathrm{~J} / \mathrm{kg} \mathrm{K})$ of PBAC [46]. The estimated temperature increase at the target surface under irradiation at $0.1 \mathrm{~J} / \mathrm{cm}^{2}$ is $1240 \mathrm{~K}$, which is much higher than the corresponding boiling points of the liquids used in this work. This implies that the formation of bubbles due to local evaporation of the solvent should be similar for all the liquids tested. In consequence, the different sizes and morphologies cannot be explained by invoking bubble formation. To this point is worth mentioning that cavitation bubbles are not visually detected. The mean diameters of the polymer nanoparticles as a function of the boiling point of the different liquids are displayed in Figure S1.

Another factor that could affect the formation of nanostructures is the density of the liquid medium. Since we worked at a fixed volume $(1 \mathrm{~mL})$, density is proportional to the mass of liquid in the vessel. Thus, a higher density implies a higher mass of liquid surrounding the target ablation site. This fact could affect the ablation plume expansion and thus the formation of nanostructures. Table 1 and Figure S2 show the mean diameter of nanoparticles in different liquid media where it becomes evident the lack of relationship between the density and particle size.

Recently, Russo et al [47] prepared copper nanoparticles by laser ablation in different gas media, such as argon (Ar) and helium (He). These authors have found that the particle size was dependent on the surrounding gas, being the mean diameters smaller for irradiation in He than in Ar. Experimental results and simulations led to conclude that the different size distributions were a consequence of the much higher thermal conductivity of He (ten times larger than Ar). Consequently, the vapor plume cools faster for ablation in He. The faster cooling rate induces a higher supersaturation ratio and a shorter condensation duration, which result in smaller particles from condensation. Also, De Bonis et al. [48] have performed PLAL experiments in different liquids, namely water and acetone, using palladium as ablation target. They found that the mean diameter of the nanoparticles obtained in acetone was slightly higher than those obtained in water under the same irradiation conditions and related these results to the thermal properties of the liquids which could affect the cooling times of the plume. Since thermal conductivity of water is larger than that of acetone, a faster cooling of the plume leads to smaller particles. Figure 9 shows the mean diameter of nanoparticles obtained by PLAL of PBAC as a function of the thermal conductivity of the liquids tested in this work. The distribution 
obtained by considering only spherical nanoparticles shows that as the thermal conductivity of the liquid increases, the nanoparticle diameter decreases. This result is in line with the reports by Russo [47] and De Bonis [48] and indicates that a faster cooling rate, induced by the liquid, leads to smaller nanoparticles, just like in a thermal quenching procedure.

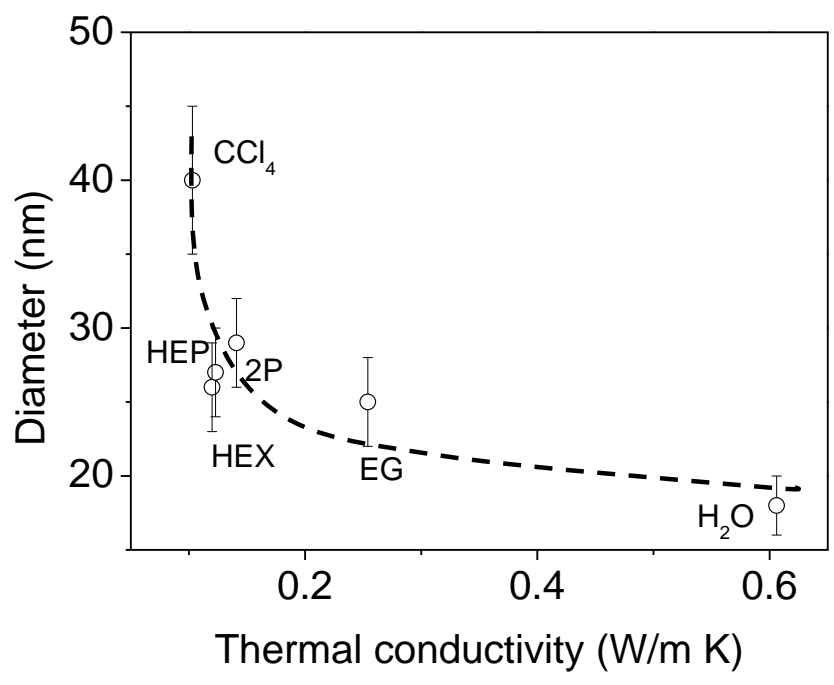

Figure 9. Mean diameter of nanoparticles as a function of the thermal conductivity of the liquid media for PLAL. The dashed line is a guide for the eye.

Additionally, the possible influence of viscosity, polarity and compressibility of the different liquids employed has been considered since they have been reported to have an effect on the nanoparticles formation in other cases [49-51]. With this purpose, the mean diameters of the nanoparticles as a function of these physical properties have been plotted in figures S3, S4 and S5, showing no clear dependence.

Regarding the different shapes of the nanostructures obtained, thermal conductivity does not completely explain all the observations and some other factors must be taken into account. In fact, the shape of the different nanostructures can be discussed in relation to the viscosity of the liquid media (Table 1). For n-hexane and heptane, which have the lowest viscosity of the studied liquids, the obtained nanoparticles are very similar in terms of size and shape and only some strands are observed in the case of irradiation in nhexane. As viscosity increases, the appearance of strands is more significant (irradiation in water and carbon tetrachloride). For even higher viscosity liquids, like 2-propanol and ethylene glycol, a new type of structure appears with a toroidal shape. These differences seem to be related to the different expansion dynamics of the ablation plume that finds a 
higher opposing force in the case of liquids with a higher viscosity. This influences the homogeneity of the ablation plume and prevents the ablated polymer nanostructures of inhomogeneous shapes to rearrange into spherical particles.

\section{Conclusions}

Pulsed laser ablation of poly(bisphenol A carbonate) was performed in different liquids leading to the formation of nanoparticles. The effect of laser fluence was first studied by irradiating the polymer in water at different fluences in the range $0.1-1 \mathrm{~J} / \mathrm{cm}^{2}$. A decrease of the size of round nanoparticles together with the appearance of strands also in the nanometer scale is observed when decreasing the irradiation fluence. For high fluences, more and faster material is ejected leading to more collisions within the ablation plume that ultimately favor formation of larger nanoparticles. The effect of the liquid media on the structures obtained is studied using liquids with different physico-chemical parameters. The different size distributions are studied as a function of density, viscosity, thermal conductivity, boiling temperature, isothermal compressibility and polarity. It is observed that they are related to the thermal properties of the liquids, which affect the cooling times of the ablation plume expanding in the liquid medium. In particular, as the thermal conductivity of the liquid increases, the faster cooling of the plume results in smaller particle size. On the other hand, the viscosity of the liquid seems to influence the development of the different nanostructures. Thus, as viscosity increases the different expansion dynamics of the ablation plume promotes new types of structures like strands and toroids.

\section{Acknowledgements}

The authors gratefully acknowledge financial support from the MINECO (CTQ 201343086-P, MAT2014-59187-R, MAT2015-66443-C02-1-R). D.E.M. thanks CSIC for the tenure of JAE-Pre fellowship and Fondo Social Europeo (FSE) for cofinancing the JAE Program. E.R. also thanks MINECO for a Ramón y Cajal contract (RYC-2011-08069). The authors thank J. V. García Ramos for the use of the UV-Vis-NIR spectrophotometer.

\section{Bibliography}


[1] C. L. Soles, Y. Ding, Nanoscale Polymer Processing, Science 322 (2008) 689-690.

[2] J. Martín, C. Mijangos, Tailored Polymer-Based Nanofibers and Nanotubes by Means of Different Infiltration Methods into Alumina Nanopores, Langmuir 25 (2008) 1181-1187.

[3] K. Landfester, The Generation of Nanoparticles in Miniemulsions, Adv. Mat. 13 (2001) 765768.

[4] D. E. Martínez-Tong, M. Soccio, A. Sanz, C. García, T. A. Ezquerra, A. Nogales, Chain Arrangement and Glass Transition Temperature Variations in Polymer Nanoparticles under 3DConfinement, Macromolecules 46 (2013) 4698-4705.

[5] G. Marcelo, J. M. G. Martinho, J. P. S. Farinha, Polymer-Coated Nanoparticles by Adsorption of Hydrophobically Modified Poly(N,N-dimethylacrylamide), J. Phys. Chem. B 117 (2013) 34163427.

[6] S. Rose, A. Prevoteau, P. Elziere, D. Hourdet, A. Marcellan, L. Leibler, Nanoparticle solutions as adhesives for gels and biological tissues, Nature 505 (2014) 382-385.

[7] N. T. D. Tran, N. P. Truong, W. Gu, Z. Jia, M. A. Cooper, M. J. Monteiro, Timed-Release Polymer Nanoparticles, Biomacromolecules 14 (2013) 495-502.

[8] T. Kietzke, D. Neher, K. Landfester, R. Montenegro, R. Guntner, U. Scherf, Novel approaches to polymer blends based on polymer nanoparticles, Nat Mater 2 (2003) 408-412.

[9] C. M. MacNeill, E. G. Graham, N. H. Levi-Polyachenko, Soft template synthesis of donoracceptor conjugated polymer nanoparticles: Structural effects, stability, and photothermal studies, J. Polym. Sci. A Polym. Chem. 52 (2014) 1622-1632.

[10] A. W. Jackson, D. A. Fulton, Making polymeric nanoparticles stimuli-responsive with dynamic covalent bonds, Polym. Chem. 4 (2013) 31-45.

[11] J. Pecher, S. Mecking, Nanoparticles of Conjugated Polymers, Chem. Rev. 110 (2010) 62606279.

[12] C. Zhang, Y. Guo, R. D. Priestley, Confined glassy properties of polymer nanoparticles, J. Polym. Sci. B Polym. Phys. 51 (2013) 574-586.

[13] D. E. Martínez-Tong, M. Soccio, A. Sanz, C. García, T. A. Ezquerra, A. Nogales, Ferroelectricity and molecular dynamics of poly(vinylidenefluoride-trifluoroethylene) nanoparticles, Polymer 56 (2015) 428-434.

[14] D. E. Martínez-Tong, J. Cui, M. Soccio, C. García, T. A. Ezquerra, A. Nogales, Does the Glass Transition of Polymers Change Upon 3D Confinement?, Macromol. Chem. Phys. 215 (2014) 1620-1624.

[15] W. M. Steen, J. Mazumder, Laser Materials Processing, Springer-Verlag, London, UK, 2010.

[16] R. Streubel, S. Barcikowski, B. Gökce, Continuous multigram nanoparticle synthesis by high-power, high-repetition-rate ultrafast laser ablation in liquids, Opt. Lett. 41 (2016) 14861489.

[17] P. P. Patil, D. M. Phase, S. A. Kulkarni, S. V. Ghaisas, S. K. Kulkarni, S. M. Kanetkar, S. B. Ogale, V. G. Bhide, Pulsed-laser induced reactive quenching at liquid-solid interface: Aqueous oxidation of iron, Phys. Rev. Lett. 58 (1987) 238-241.

[18] R. Torres La Porte, D. Silva Moreno, M. Castillejo Striano, M. Martín Muñoz, J. V. GarcíaRamos, S. Sánchez Cortés, E. Koudoumas, Laser ablation studies of deposited silver colloids active in SERS, Laser Chem. 20 (2002) 23-32.

[19] A. V. Kabashin, M. Meunier, Synthesis of colloidal nanoparticles during femtosecond laser ablation of gold in water, J. Appl. Phys. 94 (2003) 7941-7943.

[20] C. X. Wang, P. Liu, H. Cui, G. W. Yang, Nucleation and growth kinetics of nanocrystals formed upon pulsed-laser ablation in liquid, Appl. Phys. Lett. 87 (2005) -.

[21] A. V. Simakin, V. V. Voronov, N. A. Kirichenko, G. A. Shafeev, Nanoparticles produced by laser ablation of solids in liquid environment, Appl. Phys. A 79 (2004) 1127-1132.

[22] G. W. Yang, Laser ablation in liquids: Applications in the synthesis of nanocrystals, Prog. Mat. Sci. 52 (2007) 648-698.

[23] M. Cueto, M. Sanz, M. Oujja, F. Gámez, B. Martínez-Haya, M. Castillejo, Platinum Nanoparticles Prepared by Laser Ablation in Aqueous Solutions: Fabrication and Application to Laser Desorption Ionization, J. Phys. Chem. C 115 (2011) 22217-22224. 
[24] G. G. Guillen, M. I. Mendivil Palma, B. Krishnan, D. Avellaneda Avellaneda, S. Shaji, Tin sulfide nanoparticles by pulsed laser ablation in liquid, Journal of Materials Science: Materials in Electronics 27 (2016) 6859-6871.

[25] H. Wang, O. Odawara, H. Wada, One-step preparation of YVO4:Eu3+ nanoparticles by pulsed laser ablation, Journal of Alloys and Compounds 683 (2016) 1-6.

[26] Y. Tamaki, T. Asahi, H. Masuhara, Nanoparticle Formation of Vanadyl Phthalocyanine by Laser Ablation of Its Crystalline Powder in a Poor Solvent $\uparrow$, J. Phys. Chem. A 106 (2002) 21352139.

[27] Y. Tamaki, T. Asahi, H. Masuhara, Tailoring nanoparticles of aromatic and dye molecules by excimer laser irradiation, Appl. Surf. Sci. 168 (2000) 85-88.

[28] I. Elaboudi, S. Lazare, C. Belin, D. Talaga, C. Labrugère, Underwater excimer laser ablation of polymers, Appl. Phys. A 92 (2008) 743-748.

[29] A. Schwenke, P. Wagener, S. Nolte, S. Barcikowski, Influence of processing time on nanoparticle generation during picosecond-pulsed fundamental and second harmonic laser ablation of metals in tetrahydrofuran, Appl. Phys. A 104 (2011) 77-82.

[30] S. b. Besner, A. V. Kabashin, F. o. M. Winnik, M. Meunier, Synthesis of Size-Tunable Polymer-Protected Gold Nanoparticles by Femtosecond Laser-Based Ablation and Seed Growth, J. Phys. Chem. C 113 (2009) 9526-9531.

[31] M. Cueto, M. Piedrahita, C. Caro, B. Martínez-Haya, M. Sanz, M. Oujja, M. Castillejo, Platinum Nanoparticles as Photoactive Substrates for Mass Spectrometry and Spectroscopy Sensors, J. Phys. Chem. C 118 (2014) 11432-11439.

[32] CRC Handbook of Chemistry and Physics , 85th ed., CRC Press, 2003.

[33] A. Kruusing, Liquids and their properties, in Handbook of liquids-assisted laser processing (2008) 315-385.

[34] D. R. Lide, CRC Handbook of Chemistry and Physics, CRC Press, Boca Raton, 2003.

[35] C. Reichardt, Solvatochromic Dyes as Solvent Polarity Indicators, Chem. Rev. 94 (1994) 2319-2358.

[36] I. Elaboudi, S. Lazare, C. Belin, J. L. Bruneel, L. Servant, Organic nanoparticles suspensions preparation by underwater excimer laser ablation of polycarbonate, Appl. Surf. Sci. 253 (2007) 7835-7839.

[37] B. W. Muir, S. L. Mc Arthur, H. Thissen, G. P. Simon, H. J. Griesser, D. G. Castner, Effects of oxygen plasma treatment on the surface of bisphenol A polycarbonate: a study using SIMS, principal component analysis, ellipsometry, XPS and AFM nanoindentation, Surface and Interface Analysis 38 (2006) 1186-1197.

[38] E. Rebollar, M. Castillejo, T. A. Ezquerra, Laser induced periodic surface structures on polymer films: From fundamentals to applications, European Polymer Journal 73 (2015) 162174.

[39] E. Rebollar, S. Perez, M. Hernandez, C. Domingo, M. Martin, T. A. Ezquerra, J. P. GarciaRuiz, M. Castillejo, Physicochemical modifications accompanying UV laser induced surface structures on poly(ethylene terephthalate) and their effect on adhesion of mesenchymal cells, Physical Chemistry Chemical Physics 16 (2014) 17551-17559.

[40] T. Tsuji, D. H. Thang, Y. Okazaki, M. Nakanishi, Y. Tsuboi, M. Tsuji, Preparation of silver nanoparticles by laser ablation in polyvinylpyrrolidone solutions, Appl. Surf. Sci. 254 (2008) 5224-5230.

[41] G. Cristoforetti, E. Pitzalis, R. Spiniello, R. Ishak, F. Giammanco, M. Muniz-Miranda, S. Caporali, Physico-chemical properties of Pd nanoparticles produced by Pulsed Laser Ablation in different organic solvents, Appl. Surf. Sci. 258 (2012) 3289-3297.

[42] T. E. Itina, On Nanoparticle Formation by Laser Ablation in Liquids, J. Phys. Chem. C 115 (2011) 5044-5048.

[43] E. Leveugle, L. V. Zhigilei, Molecular dynamics simulation study of the ejection and transport of polymer molecules in matrix-assisted pulsed laser evaporation, J. Appl. Phys. 102 (2007) 074914.

[44] E. Leveugle, L. V. Zhigilei, A. Sellinger, J. M. Fitz-Gerald, Computational and experimental study of the cluster size distribution in MAPLE, Appl. Surf. Sci. 253 (2007) 6456-6460. 
[45] S. Lazare, V. Tokarev, A. Sionkowska, M. Wiśniewski, Surface foaming of collagen, chitosan and other biopolymer films by $\mathrm{KrF}$ excimer laser ablation in the photomechanical regime, Appl. Phys. A 81 (2005) 465-470.

[46] E. Rebollar, S. Pérez, J. J. Hernández, I. Martín-Fabiani, D. R. Rueda, T. A. Ezquerra, M. Castillejo, Assessment and Formation Mechanism of Laser-Induced Periodic Surface Structures on Polymer Spin-Coated Films in Real and Reciprocal Space, Langmuir 27 (2011) 5596-5606.

[47] S.-B. Wen, X. Mao, R. Greif, R. E. Russo, Experimental and theoretical studies of particle generation after laser ablation of copper with a background gas at atmospheric pressure, J. Appl. Phys. 101 (2007) -.

[48] A. Bonis, M. Sansone, A. Galasso, A. Santagata, R. Teghil, The role of the solvent in the ultrashort laser ablation of palladium target in liquid, Appl. Phys. A (2014) 1-6.

[49] B. Gökce, D. D. van't Zand, A. Menéndez-Manjón, S. Barcikowski, Ripening kinetics of laser-generated plasmonic nanoparticles in different solvents, Chemical Physics Letters 626 (2015) 96-101.

[50] A. Menéndez-Manjón, B. N. Chichkov, S. Barcikowski, Influence of Water Temperature on the Hydrodynamic Diameter of Gold Nanoparticles from Laser Ablation, J. Phys. Chem. C 114 (2010) 2499-2504.

[51] G. Compagnini, A. A. Scalisi, O. Puglisi, Production of gold nanoparticles by laser ablation in liquid alkanes, J. Appl. Phys. 94 (2003) 7874-7877. 